\title{
Efficient Hermite Spectral Methods for Space Tempered Fractional Diffusion Equations
}

\author{
Tengteng Cui ${ }^{1}$, Sheng Chen ${ }^{2}$ and Yujian $\mathrm{Jiao}^{3, *}$ \\ ${ }^{1}$ Fujian Provincial Key Laboratory of Mathematical Modeling and High- \\ Performance Scientific Computing and School of Mathematical Sciences, \\ Xiamen University, Xiamen 361005, P.R. China. \\ ${ }^{2}$ School of Mathematics and Statistics, Jiangsu Normal University, \\ Xuzhou 221116, P.R. China. \\ ${ }^{3}$ Department of Mathematics, Shanghai Normal University and Scientific \\ Computing Key Laboratory of Shanghai Universities, Shanghai 200234, \\ P.R. China.
}

Received 7 April 2020; Accepted (in revised version) 11 July 2020.

\begin{abstract}
Spectral and spectral collocation methods for tempered fractional diffusion equations on the real line $\mathbb{R}$ are developed. Applying the Fourier transform to the problem under consideration, we reduce it to systems of algebraic equations. Since Hermite functions are the eigenfunctions of the Fourier transform, they are used in the construction of spectral and spectral collocation methods for the algebraic equations obtained. The stability and convergence of the methods are studied. Numerical examples demonstrate the efficiency of the algorithms and confirm theoretical findings.
\end{abstract}

AMS subject classifications: 65N35, 65E05, 65M70, 41A05, 41A10, 41A25

Key words: Tempered fractional diffusion equation, Hermite functions, spectral method, spectral collocation method, problem on the whole line.

\section{Introduction}

Fractional integrals and derivatives are widely used to model anomalous phenomena arising in physics $[1,10,12,25,32-34]$, finance $[19,29,37]$, biology $[3,21]$, and hydrology $[2,13,17,31]$. A prominent example of such applications is given by the anomalous diffusion equation

$$
\partial_{t}^{\beta} p(x, t)=\partial_{x}^{\alpha} p(x, t)
$$

where $p(x, t)$ is the probability density function and $0<\beta<1,0<\alpha<2$ [32]. Since the spatial fractional derivative causes the asymptotic decay $|x|^{-1-\alpha}$ of the solution, the

${ }^{*}$ Corresponding author. Email addresses: cuitengteng@stu.xmu.edu.cn (T.T. Cui), shengchen@jsnu . edu.cn (S. Chen), yj-jiao@shnu.edu.cn (Y.J. Jiao) 
moment conditions of the traditional diffusion equation are violated. There are various approaches to fix the problem - cf. $[8,22,23,26,27,39,44]$, including the use of the exponential tempering factor $e^{-\lambda|x|}, \lambda>0$ in the particle jump density function. This leads to the tempered fractional diffusion equation, which finds numerous applications $[4-6,15$, $30,35,36]$.

In order to find the solution of a tempered fractional diffusion equation, numerical methods based on local operations have been developed recently. In particular, Li and Deng [24] considered a high order difference scheme for equations on bounded domains, Sabzikar et al. [36] introduced a finite difference method on truncated domains, Baeumera and Meerschaert [4] provided finite difference and particle tracking methods, Cartea and del-Castillo-Negrete [6] constructed a finite difference scheme for a Black-Merton-Scholes model with tempered fractional derivatives, Sun et al. [40] applied different methods to spatial operators in the time-space tempered fractional Fokker-Planck equation on a finite domain, Deng and Zhang [16] designed finite difference and finite element schemes to simulate the backward time tempered fractional Feynman-Kac equation, Dehghan and Abbaszadeh [14] employed finite element methods to the space fractional tempered diffusionwave equation, Chen and Deng [9] developed an unconditionally stable second-order finite difference scheme for the space-time tempered fractional diffusion-wave equation, Çelik and Duman [7] proposed a Galerkin finite element method for symmetric tempered fractional diffusion equations. Nevertheless, since the tempered derivative operators are global, the spectral methods using global bases are well-suited for solving tempered fractional diffusion equations. Hanert and Piret [20] employed a pseudospectral method based on the Chebyshev polynomial expansion for solving space and time tempered fractional diffusion equation. Zayernouri et al. [43] defined tempered Jacobi poly-fractonomials and used them to simulate tempered fractional differential operators. All the above mentioned numerical methods are applied to tempered fractional derivatives in the bounded domain. However, since there are many problems with tempered fractional derivatives related to random walks on the whole line, it is important to study the corresponding equations on unbounded domains. Nevertheless, there are only a few works devoted to such problems.

In this paper, we consider the following space tempered fractional diffusion equation on the real line - cf. $[5,36]$ : For $\mu \in(k-1, k), k=1,2$,

$$
\begin{aligned}
& \partial_{t} u(x, t)+(-1)^{k+1}\left(p \partial_{+, x}^{\mu, \lambda}+q \partial_{-, x}^{\mu, \lambda}\right) u(x, t)=f(x, t), \quad x \in \mathbb{R}, \quad 0<t \leq T, \\
& u(x, 0)=u_{0}(x),
\end{aligned}
$$

where $p+q=1, p, q \geq 0$, and $\partial_{+, x}^{\mu, \lambda}, \partial_{-, x}^{\mu, \lambda}$ are the tempered fractional derivatives defined by (2.5)-(2.6). Chen et al. [11] developed an efficient spectral method for solving this equation. Thus they introduced a family of generalised Laguerre functions and derived useful formulas for tempered fractional integrals and derivatives. However, the numerical implementation of their method requires substantial efforts because of the complicated definition of the tempered fractional derivatives. Recently, Mao and Shen [28] showed that the Hermite functions are the eigenfunction of the Fourier transform operator - Lemma 2.3 below. This can be used to reduce complicated non-local fractional problem to simple 
equations in the frequency space. In particular, the above tempered fractional diffusion equation is equivalent to the equation

$$
\partial_{t} \mathscr{F}[u]+(-1)^{k+1}\left\{p \mathscr{B}_{+}^{\mu, \lambda}(\xi)+q \mathscr{B}_{-}^{\mu, \lambda}(\xi)\right\} \mathscr{F}[u]=\mathscr{F}[f]
$$

where $\mathscr{F}[u]$ is the Fourier transform of $u$, and

$$
\mathscr{B}_{ \pm}^{\mu, \lambda}(\xi):= \begin{cases}(\lambda \pm \mathrm{i} \xi)^{\mu}-\lambda^{\mu}, & 0<\mu<1 \\ (\lambda \pm \mathrm{i} \xi)^{\mu}-\lambda^{\mu}- \pm \mathrm{i} \xi \mu \lambda^{\mu-1}, & 1<\mu<2\end{cases}
$$

We note that Mao and Shen [28] employed three recurrent formulas in numerical integration of the function $|\xi|^{2 s}$ arising in Fourier transform of the classical fractional Laplacian $(-\Delta)^{s}$. In our case, the Hermite quadrature formula can be directly used in order to compute the inner product involving tempered fractional derivatives. More precisely, we can accurately approximate $\left(\mathscr{B}_{ \pm}^{\mu, \lambda}(\xi) \mathscr{F}[u], \mathscr{F}[v]\right)=\left(\partial_{ \pm, x}^{\mu, \lambda} u, v\right)$ by the Hermite quadrature formula since for $\lambda>0$ the function $|\lambda \pm \mathrm{i} \xi|^{\mu}$ is smooth. Moreover, this approach has several remarkable advantages — viz.

1. By transforming the original problem into an equivalent problem in the frequency space, we can avoid the complicated tempered fractional derivatives, so that the method can be easily implemented.

2. Using the Hermite functions for the approximation of the tempered fractional diffusion equation on the whole line simplifies theoretical analysis.

3. In contrast to the fractional Laplacian [41, 42], the tempered fractional derivative leads to an exponential asymptotic behavior [36]. Hence, for smooth sources with exponential decay the methods based on the Hermite functions may yield a spectral convergence.

4. Numerical and theoretical technique discussed in this paper can be applied to other problems involving tempered fractional derivatives.

The remainder of the paper is organised as follows. In Section 2, we recall the definition and the properties of Hermite functions, tempered fractional derivatives and related approximation results. Section 3 introduces a Galerkin spectral method with Hermite functions. We prove the stability of the method and its convergence. Numerical examples confirm the theoretical analysis and validate the expected behavior of the tempered fractional diffusion. In Section 4, we consider a Hermite collocation method for tempered fractional differential equations and present numerical results, which demonstrate the efficiency of the method. The final section contains concluding remarks. 


\section{Preliminaries}

Let $\mathbb{N}$ and $\mathbb{R}$ be the sets of positive integers and real numbers, respectively. For real $f$ and $g$, the inner product and norm are defined by

$$
(f, g)_{\omega}:=\int_{\mathbb{R}} f(x) g(x) \omega(x) \mathrm{d} x, \quad\|f\|_{\omega}:=\sqrt{(f, f)_{\omega}}
$$

and the subscript $\omega$ is omitted if $\omega=1$. Let $c$ be a positive constant independent of any functions. We also write $A \lesssim B$ if $A \leq c B$.

\subsection{Hermite functions and fractional derivatives}

Let $H_{n}(x)$ denote the classical Hermite polynomial of degree $n$. Considering the corresponding Hermite functions $\psi_{n}(x)$ defined by

$$
\psi_{n}(x)=\frac{1}{\pi^{1 / 4} \sqrt{2^{n} n !}} e^{-x^{2} / 2} H_{n}(x), \quad n=0,1,2, \ldots, \quad x \in \mathbb{R},
$$

we note their following properties:

1. The functions $\psi_{n}(x)$ are mutually orthonormal - i.e.

$$
\int_{\mathbb{R}} \psi_{n}(x) \psi_{m}(x) \mathrm{d} x=\delta_{n m},
$$

where $\delta_{n m}$ is the Kronecker delta.

2. $\psi_{n}(x)$ is the $n$-th eigenfunction of the singular Sturm-Liouville problem

$$
e^{x^{2} / 2} \partial_{x}\left(e^{-x^{2}} \partial_{x}\left(e^{x^{2} / 2} u(x)\right)\right)+\lambda_{n} u(x)=0, \quad \lambda_{n}=2 n .
$$

3. The Hermite functions are generated by three-term recurrence relation

$$
\begin{aligned}
& \psi_{0}(x)=\pi^{-1 / 4} e^{-x^{2} / 2} \\
& \psi_{1}(x)=\pi^{-1 / 4} \sqrt{2} x e^{-x^{2} / 2} \\
& \psi_{n+1}(x)=\sqrt{\frac{2}{(n+1)}} x \psi_{n}(x)-\sqrt{\frac{n}{(n+1)}} \psi_{n-1}(x), \quad n \geq 1 .
\end{aligned}
$$

We next introduce a Hermite projection operator. Let $\omega(x)=e^{-x^{2}}$ and $\mathscr{P}_{N}$ be the set of polynomials of degree at most $N$. The $L_{\omega}^{2}$-orthogonal projection $\Pi_{N}: L_{w}^{2}(\mathbb{R}) \rightarrow \mathscr{P}_{N}$ is defined by

$$
\int_{\mathbb{R}}\left\{\Pi_{N} u-u\right\}(x) v_{N}(x) w(x) \mathrm{d} x=0 \quad \text { for all } \quad v_{N} \in \mathscr{P}_{N} .
$$


Consider the space

$$
\widehat{\mathscr{P}}_{N}(\mathbb{R})=\operatorname{span}\left\{\psi_{n}(x): 0 \leq n \leq N\right\}
$$

and the orthogonal projections $\widehat{\Pi}_{N}: L^{2}(\mathbb{R}) \rightarrow \widehat{\mathscr{P}}_{N}$ defined by

$$
\widehat{\Pi}_{N} u(x):=e^{-x^{2} / 2} \Pi_{N}\left(u(x) e^{x^{2} / 2}\right) .
$$

It is easily seen that for any $\psi_{n} \in \widehat{\mathscr{P}}_{N}$ one has

$$
\left(u-\widehat{\Pi}_{N} u, \psi_{n}\right)=\left(u e^{x^{2} / 2}-\Pi_{N}\left(u e^{x^{2} / 2}\right), \psi_{n} e^{x^{2} / 2}\right)_{\omega} .
$$

In order to evaluate the approximation error, we also consider the Sobolev space

$$
\widehat{B}_{r}(\mathbb{R}):=\left\{u: \widehat{\partial}_{x}^{k} u \in L^{2}(\mathbb{R}), 0 \leq k \leq r\right\},
$$

where $r \in \mathbb{N}$ and $\widehat{\partial}_{x}:=\partial_{x}+x$.

Lemma 2.1 (cf. Shen et al. [38, Theorem 7.14]). For any $u \in \widehat{B}_{r}(\mathbb{R})$ and $2 \leq r \leq N+1$,

$$
\left\|\partial_{x}^{l}\left(\widehat{\Pi}_{N} u-u\right)\right\| \lesssim N^{(l-r) / 2}\left\|\widehat{\partial}_{x}^{r} u\right\|, \quad l=0,1,2 .
$$

Let $\left\{x_{j}, \omega_{j}\right\}_{j=0}^{N}$ be the Hermite-Gauss nodes and weights. Denote by $\left\{\widehat{x}_{j}, \widehat{\omega}_{j}\right\}_{j=0}^{N}$ the Gauss quadrature nodes and weights of the Hermite function interpolation. It follows from (2.1) that $\widehat{x}_{j}=x_{j}, \widehat{\omega}_{j}=e^{\widehat{x}_{j}^{2}} \omega_{j}$. Let $I_{N}: C(\mathbb{R}) \rightarrow \mathscr{P}_{N}$ be the interpolation operator corresponding to the Hermite-Gauss nodes. The interpolation $\mathscr{I}_{N}: C(\mathbb{R}) \rightarrow \widehat{\mathscr{P}}_{N}$ associated with the Hermite functions can be defined by

$$
\mathscr{I}_{N} u=e^{-x^{2} / 2} I_{N}\left(u e^{x^{2} / 2}\right)
$$

Lemma 2.2 (cf. Shen et al. [38, Theorem 7.18]). For any $u \in C(\mathbb{R})$ and $\widehat{\partial}_{x}^{m} u \in L^{2}(\mathbb{R})$ with fixed $m \geq 1$, we have

$$
\left\|\widehat{\partial}_{x}^{k}\left(\mathscr{I}_{N} u-u\right)\right\| \lesssim N^{1 / 6+(k-m) / 2}\left\|\widehat{\partial}_{x}^{m} u\right\|, \quad 0 \leq k \leq m
$$

with the differentiation operator $\widehat{\partial}_{x}=\partial_{x}+x$.

We also employ the Fourier and inverse Fourier transforms - i.e.

$$
\begin{aligned}
& \mathscr{F}[u](\xi)=\frac{1}{\sqrt{2 \pi}} \int_{-\infty}^{\infty} u(x) e^{-\mathrm{i} \xi x} \mathrm{~d} x, \\
& u(x)=\mathscr{F}^{-1}[\mathscr{F}[u](\xi)](x)=\frac{1}{\sqrt{2 \pi}} \int_{-\infty}^{\infty} \mathscr{F}[u](\xi) e^{\mathrm{i} \xi x} \mathrm{~d} \xi,
\end{aligned}
$$

and the Parseval's identity

$$
(u, v)=\int_{-\infty}^{\infty} u(x) \overline{v(x)} \mathrm{d} x=\int_{-\infty}^{\infty} \mathscr{F}[u](\xi) \overline{\mathscr{F}[v](\xi)} \mathrm{d} \xi,
$$

where $\overline{v(x)}$ is the complex conjugate of the function $v(x)$ [18]. 
Lemma 2.3. The Hermite functions $\psi_{n}, n=0,1, \ldots$ are the eigenfunctions of the Fourier operator $\mathscr{F}$ corresponding the eigenvalues $(-\mathrm{i})^{n}$, i.e.

$$
\mathscr{F}\left[\psi_{n}\right](\xi)=(-\mathrm{i})^{n} \psi_{n}(\xi), \quad \mathrm{i}=\sqrt{-1} .
$$

We next consider the tempered fractional derivatives. According to [36], the left and right tempered fractional derivatives of a function $u(x)$ of order $\mu$ are defined in the following way:

1. If $0<\mu<1$, then

$$
\begin{aligned}
& \partial_{+, x}^{\mu, \lambda} u(x):=\frac{\mu}{\Gamma(1-\mu)} \int_{0}^{\infty}(u(x)-u(x-t)) e^{-\lambda t} t^{-\mu-1} \mathrm{~d} t \\
& \partial_{-, x}^{\mu, \lambda} u(x)=\frac{\mu}{\Gamma(1-\mu)} \int_{0}^{\infty}(u(x)-u(x+t)) e^{-\lambda t} t^{-\mu-1} \mathrm{~d} t .
\end{aligned}
$$

2. If $1<\mu<2$, then

$$
\begin{aligned}
& \partial_{+, x}^{\mu, \lambda} u(x)=\frac{\mu(\mu-1)}{\Gamma(2-\mu)} \int_{0}^{\infty}\left(u(x-t)-u(x)+t u^{\prime}(x)\right) e^{-\lambda t} t^{-\mu-1} \mathrm{~d} t \\
& \partial_{-, x}^{\mu, \lambda} u(x)=\frac{\mu(\mu-1)}{\Gamma(2-\mu)} \int_{0}^{\infty}\left(u(x+t)-u(x)-t u^{\prime}(x)\right) e^{-\lambda t} t^{-\mu-1} \mathrm{~d} t .
\end{aligned}
$$

Applying the Fourier transform to the tempered fractional derivatives above yields

$$
\mathscr{F}\left[\partial_{ \pm, x}^{\mu, \lambda} u\right](\xi)= \begin{cases}\left((\lambda \pm \mathrm{i} \xi)^{\mu}-\lambda^{\mu}\right) \mathscr{F}[u](\xi), & 0<\mu<1, \\ \left((\lambda \pm \mathrm{i} \xi)^{\mu}-\lambda^{\mu} \mp \mathrm{i} \xi \mu \lambda^{\mu-1}\right) \mathscr{F}[u](\xi), & 1<\mu<2 .\end{cases}
$$

Lemma 2.4. Let $p+q=1, p, q \geq 0$ and $\mu \in(k-1, k), k=1,2$. For any $u \neq 0$, the tempered fractional derivatives (2.5)-(2.6) satisfy the relations

$$
\left(\mathscr{A}_{p, q}^{\mu, \lambda} u, u\right)>0, \quad \mathscr{A}_{p, q}^{\mu, \lambda} u=(-1)^{k+1}\left(p \partial_{+, x}^{\mu, \lambda}+q \partial_{-, x}^{\mu, \lambda}\right) u .
$$

Proof. For notational simplicity, we set

$$
\mathscr{B}_{p, q}^{\mu, \lambda}(\xi):=(-1)^{k+1}\left\{p \mathscr{B}_{+}^{\mu, \lambda}(\xi)+q \mathscr{B}_{-}^{\mu, \lambda}(\xi)\right\},
$$

where

$$
\mathscr{B}_{ \pm}^{\mu, \lambda}(\xi):= \begin{cases}(\lambda \pm \mathrm{i} \xi)^{\mu}-\lambda^{\mu}, & 0<\mu<1, \\ (\lambda \pm \mathrm{i} \xi)^{\mu}-\lambda^{\mu}-\left( \pm \mathrm{i} \xi \mu \lambda^{\mu-1}\right), & 1<\mu<2 .\end{cases}
$$

The Parseval identity (2.4) and representations (2.7) give

$$
\left(\mathscr{B}_{p, q}^{\mu, \lambda}(\xi) \mathscr{F}[u], \mathscr{F}[u]\right)=\left(\mathscr{A}_{p, q}^{\mu, \lambda} u, u\right) .
$$

Moreover, the equations

$$
\overline{\mathscr{F}[u](\xi)}=\mathscr{F}[u](-\xi), \quad|\mathscr{F}[u](\xi)|^{2}=|\mathscr{F}[u](-\xi)|^{2}
$$

yield: 
1. If $0<\mu<1$, then

$$
\begin{aligned}
& \left(\mathscr{B}_{p, q}^{\mu, \lambda}(\xi) \mathscr{F}[u], \mathscr{F}[u]\right) \\
= & \int_{0}^{+\infty} \mathscr{B}_{p, q}^{\mu, \lambda}(\xi)|\mathscr{F}[u](\xi)|^{2} \mathrm{~d} \xi+\int_{-\infty}^{0} \mathscr{B}_{p, q}^{\mu, \lambda}(\xi)|\mathscr{F}[u](\xi)|^{2} \mathrm{~d} \xi \\
= & \int_{0}^{+\infty}\left[p(\lambda+\mathrm{i} \xi)^{\mu}+q(\lambda-\mathrm{i} \xi)^{\mu}-\lambda^{\mu}\right]|\mathscr{F}[u](\xi)|^{2} \mathrm{~d} \xi \\
& +\int_{-\infty}^{0}\left[p(\lambda+\mathrm{i} \xi)^{\mu}+q(\lambda-\mathrm{i} \xi)^{\mu}-\lambda^{\mu}\right]|\mathscr{F}[u](\xi)|^{2} \mathrm{~d} \xi \\
= & \int_{0}^{+\infty}\left[(\lambda+\mathrm{i} \xi)^{\mu}+(\lambda-\mathrm{i} \xi)^{\mu}-2 \lambda^{\mu}\right]|\mathscr{F}[u](\xi)|^{2} \mathrm{~d} \xi .
\end{aligned}
$$

2. If $1<\mu<2$, then

$$
\begin{aligned}
& \left(\mathscr{B}_{p, q}^{\mu, \lambda}(\xi) \mathscr{F}[u], \mathscr{F}[u]\right) \\
= & \int_{0}^{+\infty} \mathscr{B}_{p, q}^{\mu, \lambda}(\xi)|\mathscr{F}[u](\xi)|^{2} \mathrm{~d} \xi+\int_{-\infty}^{0} \mathscr{B}_{p, q}^{\mu, \lambda}(\xi)|\mathscr{F}[u](\xi)|^{2} \mathrm{~d} \xi \\
= & -\int_{0}^{+\infty}\left[p(\lambda+\mathrm{i} \xi)^{\mu}+q(\lambda-\mathrm{i} \xi)^{\mu}-(p-q) \mathrm{i} \xi \mu \lambda^{\mu-1}-\lambda^{\mu}\right]|\mathscr{F}[u](\xi)|^{2} \mathrm{~d} \xi \\
& -\int_{-\infty}^{0}\left[p(\lambda+\mathrm{i} \xi)^{\mu}+q(\lambda-\mathrm{i} \xi)^{\mu}-(p-q) \mathrm{i} \xi \mu \lambda^{\mu-1}-\lambda^{\mu}\right]|\mathscr{F}[u](\xi)|^{2} \mathrm{~d} \xi \\
= & \int_{0}^{+\infty}\left[2 \lambda^{\mu}-(\lambda+\mathrm{i} \xi)^{\mu}-(\lambda-\mathrm{i} \xi)^{\mu}\right]|\mathscr{F}[u](\xi)|^{2} \mathrm{~d} \xi .
\end{aligned}
$$

Therefore,

$$
\left(\mathscr{A}_{p, q}^{\mu, \lambda} u, u\right)=\int_{0}^{+\infty}(-1)^{k+1}\left[(\lambda+\mathrm{i} \xi)^{\mu}+(\lambda-\mathrm{i} \xi)^{\mu}-2 \lambda^{\mu}\right]|\mathscr{F}[u](\xi)|^{2} \mathrm{~d} \xi>0,
$$

since $\mathscr{F}[u](\xi) \neq 0$ and $(-1)^{k+1}\left[(\lambda+\mathrm{i} \xi)^{\mu}+(\lambda-\mathrm{i} \xi)^{\mu}-2 \lambda^{\mu}\right]>0$ for $\xi \neq 0$. The latter inequality can be proved similar to the proof of [11, theorem 5.1].

\subsection{Tempered fractional Hilbert space}

Using the positivity of the inner product $\left(\mathscr{A}_{p, q}^{\mu, \lambda} u, u\right)$, we can consider the fractional Hilbert space

$$
H_{p, q}^{s, \lambda}(\mathbb{R})=\left\{v \in L^{2}(\mathbb{R}):\left(\mathscr{A}_{p, q}^{2 s, \lambda} v, v\right)<\infty\right\}, \quad s \in(0,1)
$$


and equip it with the following norm and semi-norm:

$$
\|v\|_{H_{p, q}^{s, \lambda}}=\sqrt{(v, v)+\left(\mathscr{A}_{p, q}^{2 s, \lambda} v, v\right)}, \quad|v|_{H_{p, q}^{s, \lambda}}=\sqrt{\left(\mathscr{A}_{p, q}^{2 s, \lambda} v, v\right)} .
$$

Lemma 2.5 (Interpolation Inequality). For any $\partial_{x}^{l} v \in L^{2}(\mathbb{R}), l=0,1$, we have

$$
\|v\|_{H_{p, q}^{s, \lambda}} \leq \sqrt{\|v\|^{2(1-s)}\left(\lambda^{2}\|v\|^{2}+\left\|\partial_{x} v\right\|^{2}\right)^{s}+\left(1+\lambda^{2 s}\right)\|v\|^{2}}
$$

where $0<s<1$.

Proof. Using the Hölder inequality and the identities

$$
\begin{aligned}
& |\mathscr{F}[v](\xi)|^{2}=|\mathscr{F}[v](-\xi)|^{2}, \\
& \left\|\partial_{x} v\right\|^{2}=\int_{-\infty}^{+\infty} \xi^{2}|\mathscr{F}[v](\xi)|^{2} \mathrm{~d} \xi=2 \int_{0}^{+\infty} \xi^{2}|\mathscr{F}[v](\xi)|^{2} \mathrm{~d} \xi,
\end{aligned}
$$

we obtain

$$
\begin{aligned}
& \int_{0}^{+\infty}\left[(\lambda+\mathrm{i} \xi)^{2 s}+(\lambda-\mathrm{i} \xi)^{2 s}\right]|\mathscr{F}[v](\xi)|^{2} \mathrm{~d} \xi \\
\leq & 2 \int_{0}^{+\infty}\left(\lambda^{2}+\xi^{2}\right)^{s}|\mathscr{F}[v](\xi)|^{2} \mathrm{~d} \xi \\
\leq & \left(2 \int_{0}^{+\infty}|\mathscr{F}[v](\xi)|^{2} \mathrm{~d} \xi\right)^{1-s}\left(2 \int_{0}^{+\infty}\left(\lambda^{2}+\xi^{2}\right)|\mathscr{F}[v](\xi)|^{2} \mathrm{~d} \xi\right)^{s} \\
= & \|v\|^{2-2 s}\left(\sqrt{\lambda^{2}\|v\|^{2}+\left\|\partial_{x} v\right\|^{2}}\right)^{2 s} .
\end{aligned}
$$

The proof can be completed by using the relation

$$
\begin{aligned}
\|v\|_{H_{p, q}^{s, \lambda}}^{2} & =(v, v)+\left(\mathscr{A}_{p, q}^{2 s, \lambda} v, v\right)=\|v\|^{2}+\left(\mathscr{B}_{p, q}^{2 s, \lambda}(\xi) \mathscr{F}[v], \mathscr{F}[v]\right) \\
& =\|v\|^{2}+\int_{0}^{+\infty}(-1)^{k+1}\left[(\lambda+\mathrm{i} \xi)^{2 s}+(\lambda-\mathrm{i} \xi)^{2 s}-2 \lambda^{2 s}\right]|\mathscr{F}[v](\xi)|^{2} \mathrm{~d} \xi,
\end{aligned}
$$

where $\mathscr{B}_{p, q}^{2 s, \lambda}(\xi)$ is defined in (2.9).

Theorem 2.1. Let $s \in(0,1)$. For any $v \in \widehat{B}_{r}(\mathbb{R})$ and $1 \leq r \leq N+1$, the following inequality holds:

$$
\left\|\widehat{\Pi}_{N} v-v\right\|_{H_{p, q}^{s, \lambda}} \lesssim N^{(s-r) / 2}\left\|\widehat{\partial}_{x}^{r} v\right\|,
$$

where $\widehat{\partial}_{x}=\partial_{x}+x$ and $\widehat{\Pi}$ is the projection operator (2.2).

Proof. The proof follows from Lemmas 2.1 and 2.5. 
Theorem 2.2. Let $\mu \in[0,2]$. For any $v \in \widehat{B}_{r}(\mathbb{R})$ and $2 \leq r \leq N+1$, we have

$$
\left\|\mathscr{A}_{p, q}^{\mu, \lambda}\left\{\widehat{\Pi}_{N} v-v\right\}\right\| \lesssim N^{(\mu-r) / 2}\left\|\widehat{\partial}_{x}^{r} v\right\|
$$

where $\mathscr{A}_{p, q}^{\mu, \lambda}$ is the operator defined in (2.8).

Proof. Let $\Theta(\xi) \in(-\pi / 2, \pi / 2)$ be the argument of $\lambda+\mathrm{i} \xi$, i.e.

$$
\Theta(\xi)= \begin{cases}\arccos \left(\frac{\lambda}{\sqrt{\lambda^{2}+\xi^{2}}}\right), & \text { if } \quad \xi>0, \\ -\arccos \left(\frac{\lambda}{\sqrt{\lambda^{2}+\xi^{2}}}\right), & \text { if } \quad \xi<0 .\end{cases}
$$

Since the cases $0<\mu<1$ and $1<\mu<2$ are similar, we only consider the later one. It follows from (2.9) that for $1<\mu<2$ one has

$$
\mathscr{B}_{p, q}^{\mu, \lambda}(\xi)=\lambda^{\mu}-\left(\xi^{2}+\lambda^{2}\right)^{\mu / 2} \cos (\mu \Theta)+\mathrm{i}(p-q)\left(\left(\xi^{2}+\lambda^{2}\right)^{\mu / 2} \sin (\mu \Theta)+\xi \mu \lambda^{\mu-1}\right),
$$

and

$$
\begin{aligned}
& \left(\lambda^{\mu}-\left(\xi^{2}+\lambda^{2}\right)^{\mu / 2} \cos (\mu \Theta)\right)^{2} \leq 2 \lambda^{2 \mu}+2\left(\xi^{2}+\lambda^{2}\right)^{\mu}, \\
& {\left[(p-q)\left(\left(\xi^{2}+\lambda^{2}\right)^{\mu / 2} \sin (\mu \Theta)+\xi \mu \lambda^{\mu-1}\right)\right]^{2} \leq(p-q)^{2}\left(2\left(\xi^{2}+\lambda^{2}\right)^{\mu}+2 \xi^{2} \mu^{2} \lambda^{2 \mu-2}\right) .}
\end{aligned}
$$

The above formulas, the Parseval identity and the Hölder inequality give

$$
\begin{aligned}
\left\|A_{p, q}^{\mu, \lambda} v\right\|^{2}= & \left(\mathscr{B}_{p, q}^{\mu, \lambda}(\xi) \mathscr{F}[v], \mathscr{B}_{p, q}^{\mu, \lambda}(\xi) \mathscr{F}[v]\right)=\int_{\mathbb{R}}\left|\mathscr{B}_{p, q}^{\mu, \lambda}(\xi)\right|^{2}|\mathscr{F}[v](\xi)|^{2} \mathrm{~d} \xi \\
\leq & 2 \lambda^{2 \mu}\|v\|^{2}+2\left(1+(p-q)^{2}\right) \int_{\mathbb{R}}\left(\xi^{2}+\lambda^{2}\right)^{\mu}|\mathscr{F}[v](\xi)|^{2} \mathrm{~d} \xi \\
& +2(p-q)^{2} \mu^{2} \lambda^{2 \mu-2} \int_{\mathbb{R}} \xi^{2}|\mathscr{F}[v](\xi)|^{2} \mathrm{~d} \xi \\
\leq & 2 \lambda^{2 \mu}\|v\|^{2}+4\|v\|^{2-2 \mu}\left(\lambda^{2}\|v\|^{2}+\left\|\partial_{x} v\right\|^{2}\right)^{\mu}+2 \mu^{2} \lambda^{2 \mu-2}\left\|\partial_{x} v\right\|^{2} .
\end{aligned}
$$

Combining (2.11) and Lemma 2.1 finishes the proof.

\section{Hermite Spectral Methods}

In this section, we construct an efficient Hermite Galerkin spectral method for the tempered fractional diffusion equation

$$
\begin{aligned}
& \partial_{t} u(x, t)+(-1)^{k+1}\left(p \partial_{+, x}^{\mu, \lambda}+q \partial_{-, x}^{\mu, \lambda}\right) u(x, t)=f(x, t), \\
& u(x, 0)=u_{0}(x), \quad \lim _{|x| \rightarrow+\infty} u(x, t)=0, \quad x \in \mathbb{R}, \quad 0<t \leq T .
\end{aligned}
$$




\subsection{Weak formulation and spectral scheme}

The weak formulation for (3.1) is to find $u(\cdot, t) \in H_{p, q}^{\mu / 2, \lambda}(\mathbb{R}), 0 \leq t \leq T$ such that

$$
\begin{aligned}
& \left(\partial_{t} u(\cdot, t), v\right)+\left(\mathscr{A}_{p, q}^{\mu, \lambda} u(\cdot, t), v\right)=(f(\cdot, t), v), \\
& (u(\cdot, 0), v)=\left(u_{0}, v\right) \text { for all } v \in H_{p, q}^{\mu / 2, \lambda}(\mathbb{R}) .
\end{aligned}
$$

The well-posedness of the problem (3.1) can be checked by choosing $v=2 u$. In fact, the inequality

$$
\partial_{t}\|u(\cdot, t)\|^{2}+2|u(\cdot, t)|_{H_{p, q}^{\mu / 2, \lambda}}^{2} \leq\|f(\cdot, t)\|^{2}+\|u(\cdot, t)\|^{2}
$$

holds with $|u(\cdot, t)|_{H_{p, q}^{\mu / 2, \lambda}}$ defined in (2.10). Setting

$$
E(u, t)=\|u(\cdot, t)\|^{2}+2 \int_{0}^{t}|u(\cdot, s)|_{H_{p, q}^{\mu / 2, \lambda}}^{2} \mathrm{~d} s,
$$

and using (3.3)-(3.4), we obtain

$$
\partial_{t} E(u, t) \leq\|f(\cdot, t)\|^{2}+\|u(\cdot, t)\|^{2} \leq\|f(\cdot, t)\|^{2}+E(u, t) .
$$

This yields the inequality

$$
\partial_{t}\left[e^{-t} E(u, t)\right] \leq e^{-t}\|f(\cdot, t)\|^{2},
$$

and its integral version

$$
E(u, t) \leq e^{t}\left\{\left\|u_{0}\right\|+\int_{0}^{t} e^{-s}\|f(\cdot, s)\|^{2} d s\right\} .
$$

The Galerkin method for the problem (3.2) consists in finding $u_{N}(\cdot, t) \in \widehat{\mathscr{P}}_{N}, 0 \leq t \leq T$ such that

$$
\begin{aligned}
& \left(\partial_{t} u_{N}(\cdot, t), v_{N}\right)+\left(\mathscr{A}_{p, q}^{\mu, \lambda} u_{N}(\cdot, t), v_{N}\right)=\left(\mathscr{I}_{N} f(\cdot, t), v_{N}\right), \\
& \left(u_{N}(\cdot, 0), v_{N}\right)=\left(\widehat{\Pi}_{N} u_{0}, v_{N}\right) \text { for all } v_{N} \in \widehat{\mathscr{P}}_{N} .
\end{aligned}
$$

Following the considerations in (3.3)-(3.5), one obtains

$$
E\left(u_{N}, t\right) \leq e^{t}\left(\left\|\widehat{\Pi}_{N} u_{0}\right\|+\int_{0}^{t} e^{-s}\left\|\mathscr{I}_{N} f(\cdot, s)\right\|^{2} d s\right) .
$$

\subsection{Numerical implementation}

For a fixed $t \in(0, T]$, the approximate solution $u_{N}$ is sought in the form

$$
u_{N}(x, t)=\sum_{l=0}^{N} \tilde{u}_{l}(t) \psi_{l}(x) .
$$


Substituting $u_{N}$ and $v_{N}=\psi_{m}(x), m=0,1, \ldots, N$ into (3.6) leads to the system of the first order ordinary differential equations

$$
\mathbf{M U}_{t}(t)+\mathbf{S U}(t)=\mathbf{F}(t)
$$

with the vectors

$$
\mathbf{U}(t)=\left(\tilde{u}_{0}(t), \cdots, \tilde{u}_{N}(t)\right)^{T}, \quad \mathbf{F}(t)=\left(\left(\mathscr{I}_{N} f(t), \psi_{0}\right), \cdots,\left(\mathscr{I}_{N} f(t), \psi_{N}\right)\right)^{T}
$$

and the matrices

$$
\begin{aligned}
\mathbf{M}=\left(a_{m l}\right), & a_{m l}=\left(\psi_{l}, \psi_{m}\right)=\delta_{m l}, \\
\mathbf{S}=\left(b_{m l}\right), & b_{m l}=\left(\mathscr{A}_{p, q}^{\mu, \lambda} \psi_{l}, \psi_{m}\right)=\left(\mathscr{B}_{p, q}^{\mu, \lambda} \mathscr{F}\left[\psi_{l}\right], \mathscr{F}\left[\psi_{m}\right]\right), \quad 0 \leq m, l \leq N .
\end{aligned}
$$

According to Lemma 2.3, the entries $b_{m l}$ can be calculated as

$$
\begin{aligned}
b_{m l} & =\left(\mathscr{B}_{p, q}^{\mu, \lambda} \mathscr{F}\left[\psi_{l}\right], \mathscr{F}\left[\psi_{m}\right]\right)=(-\mathrm{i})^{l} \cdot(\overline{-\mathrm{i}})^{m} \int_{-\infty}^{\infty} \mathscr{B}_{p, q}^{\mu, \lambda}(\xi) \psi_{l}(\xi) \overline{\psi_{m}(\xi)} \mathrm{d} \xi \\
& \approx(-\mathrm{i})^{l} \cdot(\overline{-\mathrm{i}})^{m} \sum_{j=0}^{N} \mathscr{B}_{p, q}^{\mu, \lambda}\left(\widehat{x}_{j}\right) \psi_{l}\left(\widehat{x}_{j}\right) \psi_{m}\left(\widehat{x}_{j}\right) \widehat{\omega}_{j},
\end{aligned}
$$

where $\left\{\widehat{x}_{j}\right\}_{j=0}^{N}$ and $\left\{\widehat{\omega}_{j}\right\}_{j=0}^{N}$ are, respectively, Gauss quadrature nodes and weights of the Hermite function interpolation and $\mathscr{B}_{p, q}^{\mu, \lambda}(\xi)$ are defined in (2.9).

Remark 3.1. Recently, Mao and Shen [28] employed Hermite functions to approximate the fractional Laplacian $(-\Delta)^{s}, 0<s<1$ on unbounded domains. In particular, they used a three recurrence formula to compute the entries

$$
s_{l m}=\int_{-\infty}^{\infty}|\xi|^{2 s} \psi_{m}(\xi) \psi_{l}(\xi) \mathrm{d} \xi
$$

of the corresponding stiffness matrix. In tempered fractional diffusion equation, the integrand $\mathscr{B}_{p, q}^{\mu, \lambda}(\xi) e^{-\xi^{2}}$ is smooth rather than the singular term $|\xi|^{2 s}$. So the stiffness matrix $\mathbf{S}$ in (3.8) can be directly computed by traditional Hermite-Gauss quadratures.

We note that the system of the first order ordinary differential equations (3.8) can be solved by various numerical methods, including Euler, Runge-Kutta or Crank-Nicolson method.

\subsection{Convergence analysis}

Using the notation $U_{N}^{*}=\widehat{\Pi}_{N} u$, we rewrite (3.2) as

$$
\left(\partial_{t} U_{N}^{*}, v_{N}\right)+\left(\mathscr{A}_{p, q}^{\mu, \lambda} U_{N}^{*}, v_{N}\right)=\left(f, v_{N}\right)+\left(\partial_{t}\left\{U_{N}^{*}-u\right\}, v_{N}\right)+\left(\mathscr{A}_{p, q}^{\mu, \lambda}\left\{U_{N}^{*}-u\right\}, v_{N}\right) .
$$

For notational simplicity, we further denote $\tilde{e}_{N}=U_{N}^{*}-u_{N}, \tilde{f}_{N}=f-\mathscr{I}_{N} f$, and 


$$
F\left(u, f, U_{N}^{*}\right)=f+\partial_{t}\left\{U_{N}^{*}-u\right\}+\mathscr{A}_{p, q}^{\mu, \lambda}\left\{U_{N}^{*}-u\right\} .
$$

Subtracting (3.6) from (3.9) yields

$$
\left(\partial_{t} \tilde{e}_{N}, v_{N}\right)+\left(\mathscr{A}_{p, q}^{\mu, \lambda} \tilde{e}_{N}, v_{N}\right)=\left(F\left(u, \tilde{f}_{N}, U_{N}^{*}\right), v_{N}\right), \quad\left(\tilde{e}_{N}, v_{N}\right)=0 \text { for all } v_{N} \in \widehat{\mathscr{P}}_{N} .
$$

Choosing $v_{N}=2 \tilde{e}_{N}$ and using (3.7), we obtain

$$
E\left(\tilde{e}_{N}, t\right) \leq e^{t} \int_{0}^{t} e^{-s}\left\|F\left(u, \tilde{f}_{N}, U_{N}^{*}\right)\right\|^{2} d s,
$$

and the triangle inequality implies

$$
\left\|F\left(u, \tilde{f}_{N}, U_{N}^{*}\right)\right\| \leq\left\|f-\mathscr{I}_{N} f\right\|+\left\|\partial_{t}\left(U_{N}^{*}-u\right)\right\|+\left\|\mathscr{A}_{p, q}^{\mu, \lambda}\left(U_{N}^{*}-u\right)\right\| .
$$

Theorem 3.1. Let $u$ and $u_{N}$ be the solutions of (3.2) and (3.6), respectively. If $f \in$ $L^{2}\left(0, T ; \widehat{B}_{m}(\mathbb{R})\right)$ and $u \in H^{1}\left(0, T ; L^{2}(\mathbb{R})\right) \cap H^{1}\left(0, T ; \widehat{B}_{r}(\mathbb{R})\right) \cap L^{2}\left(0, T ; \widehat{B}_{r}(\mathbb{R})\right)$ for a given $\lambda>0$, then the estimate

$$
\left\|u(\cdot, t)-u_{N}(\cdot, t)\right\| \lesssim \sqrt{N^{1 / 3-m}+N^{\mu-r}}\left(e^{t} \int_{0}^{t} e^{-s} V_{m, r}\{u(\cdot, s)\} \mathrm{d} s\right)
$$

holds with

$$
V_{m, r}\{u(\cdot, t)\}=\sqrt{\left\|\widehat{\partial}_{x}^{m} f(\cdot, t)\right\|^{2}+\left\|\widehat{\partial}_{x}^{r} \partial_{t} u(\cdot, t)\right\|^{2}+\left\|\widehat{\partial}_{x}^{r} u(\cdot, t)\right\|^{2}} .
$$

Proof. Applying Lemmas 2.1 and 2.2 with $k=0$ gives

$$
\left\|f-\mathscr{I}_{N} f\right\| \lesssim N^{1 / 6-m / 2}\left\|\widehat{\partial}_{x}^{m} f\right\|, \quad\left\|\partial_{t}\left(U_{N}^{*}-u\right)\right\| \lesssim N^{-r / 2}\left\|\widehat{\partial}_{x}^{r} \partial_{t} u\right\| .
$$

The term $\left\|\mathscr{A}_{p, q}^{\mu, \lambda}\left(U_{N}^{*}-u\right)\right\|$ can be estimated by Theorem 2.2 , so that

$$
\left\|\mathscr{A}_{p, q}^{\mu, \lambda}\left(U_{N}^{*}-u\right)\right\| \lesssim N^{(\mu-r) / 2}\left\|\widehat{\partial}_{x}^{r} u\right\| .
$$

The estimate (3.11) is now follows from (3.10)-(3.12) and the triangle inequality

$$
\left\|u-u_{N}\right\| \leq\left\|u-U_{N}^{*}\right\|+\left\|\tilde{e}_{N}\right\| \leq\left\|u-U_{N}^{*}\right\|+\sqrt{E\left(\tilde{e}_{N}, t\right)} .
$$

\subsection{Numerical examples}

In the forthcoming examples, the Crank-Nicolson scheme is used to solve the system of first order ordinary differential equations (3.8). In order to compute $\mathbf{M}, \mathbf{S}$ and $\mathbf{F}$, the Hermite Gauss quadrature with the degree of freedom $N+1$ is employed. 
Example 3.1. In the Eq. (3.1) we choose the parameters $p=q=1 / 2, \lambda=5 / 2$, forcing term $f(x, t)=\cos (t) e^{-x^{2}}$ and the initial distribution $u_{0}=10 e^{-4 x^{2}}$.

With fixed time step $\tau=0.001$ and $T=1$, we plot the $L^{\infty}$-error $\max _{x}\left(\mid u_{N}^{\tau}(x, T)-\right.$ $u(x, T) \mid)$ on the left of Fig. 1, and for fixed $\mu=1.7$ we draw the errors with distinct $\tau$ on the right of Fig. 1. Since the exact solution of the problem is not known, the term $u=u_{N}^{\tau}(x, T), \tau=10^{-4}, N=150$ is used as the reference solution in both cases. Numerical results demonstrate the efficiency of the method in the approximation of tempered fractional diffusion equations.
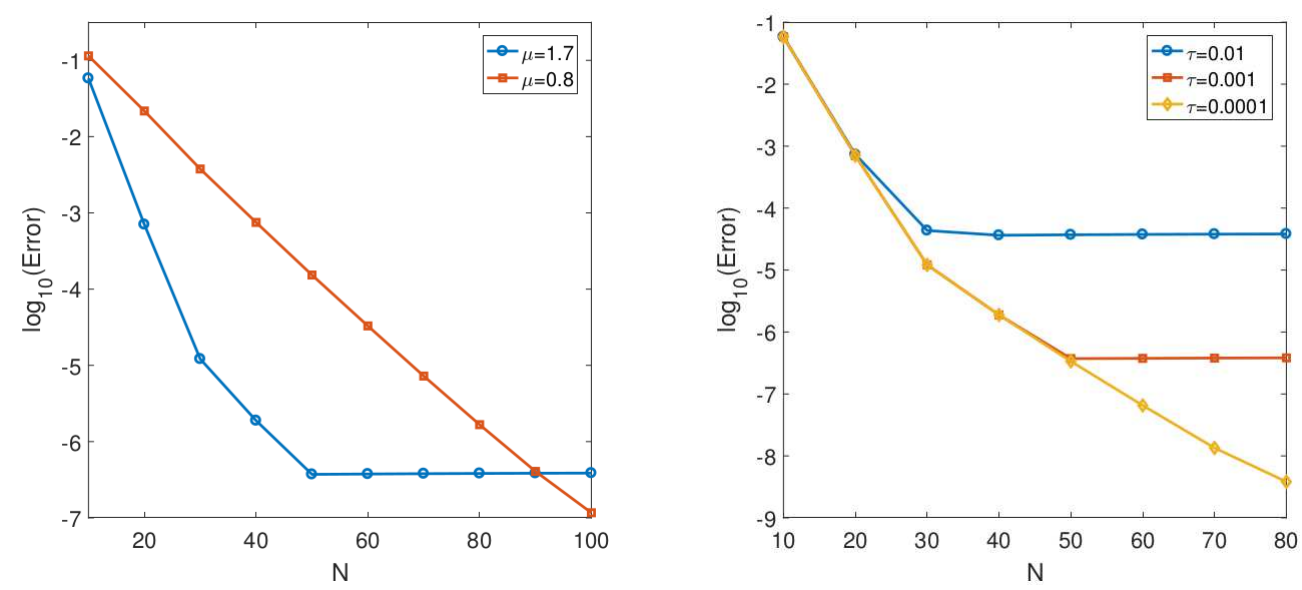

Figure 1: Left: $\mu=0.8,1.7$ with $\tau=0.001$. Right: $\mu=1.7$ with distinct $\tau$.

Example 3.2. Consider the tempered fractional diffusion (3.1) with $u_{0}=10 e^{-4 x^{2}}$ and $f(x, t)=0$. We simulate the diffusion process for understanding the roles of the parameters $p, q$ and $\lambda$.

Fig. 2 demonstrates the solution profile at different time with $p=1 / 4, q=3 / 4, \mu=$ $0.9, \lambda=2$ (left) and $p=3 / 4, q=1 / 4, \mu=0.9, \lambda=2$ (right), respectively. The case $p=q=1 / 2$ is presented in Fig. 3 . We note that for $p>q$ the particles tend to jump to the right, and for $p<q$ they tend to jump to the left. If $p=q$ the probabilities of particles jumping are equal. In order to make comparison with usual fractional diffusion equation, i.e. if $\lambda=0$, on the right of Fig. 3 we plot the corresponding distributions at time $t=10$. It shows that for large $|x|$ the tail of the tempered fractional diffusion behaves as $|x|^{-\mu-1} e^{-\lambda|x|}$ and the usual fractional diffusion behaves as $|x|^{-\mu-1}$.

\section{Hermite Spectral Collocation Methods}

Considering the images of the Hermite functions under Fourier transform, one can develop an efficient Hermite collocation method for tempered fractional differential equations. Let us first consider the steady tempered fractional differential equation

$$
u(x)+(-1)^{k+1}\left\{p \partial_{+, x}^{\mu, \lambda}+q \partial_{-, x}^{\mu, \lambda}\right\} u(x)=f(x), \quad \mu \in(k-1, k), \quad k=1,2,
$$



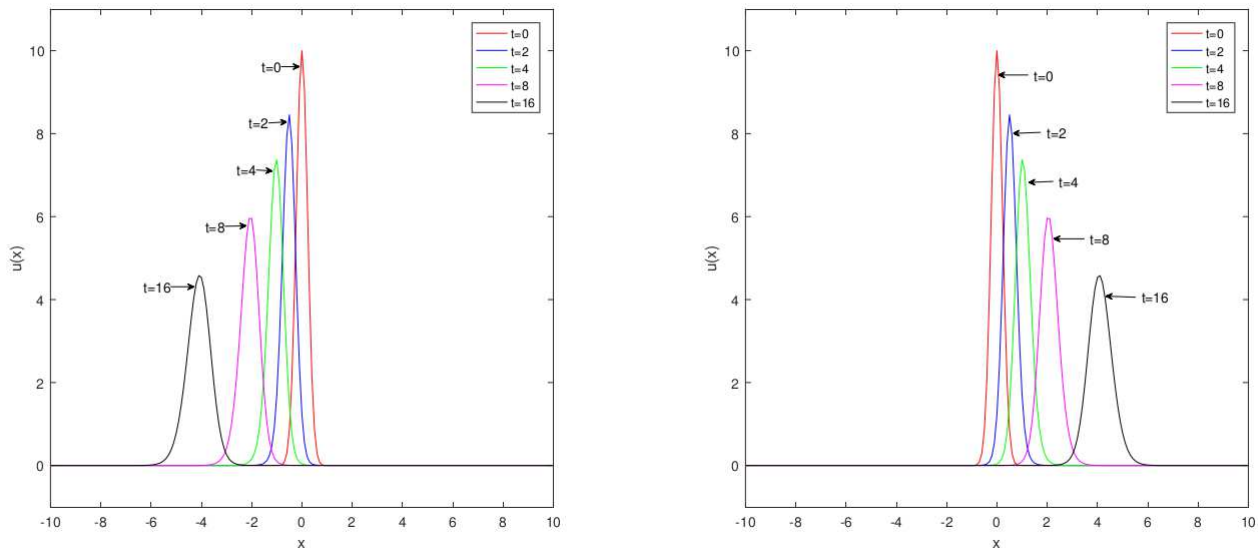

Figure 2: Left: $p=1 / 4, q=3 / 4, \lambda=2$. Right: $p=3 / 4, q=1 / 4, \lambda=2$.
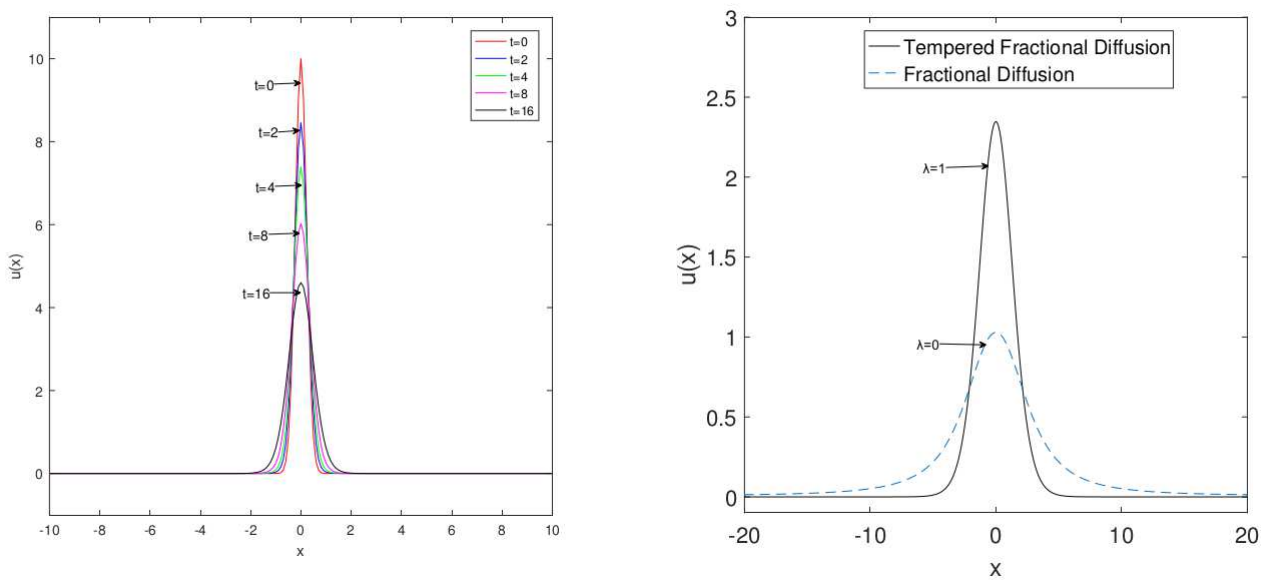

Figure 3: Left: $p=q=1 / 2, \lambda=2$. Right: $p=q$ with $\lambda=1$ and $\lambda=0$, respectively.

where $p, q$ are nonnegative constants such that $p+q=1, f \in L^{2}(\mathbb{R})$, and $\lambda>0$ is a tempered parameter. Applying the Fourier transform (2.7), we reduce the problem (4.1) to the following equation:

$$
\mathscr{F}[u](\xi)+\mathscr{B}_{p, q}^{\mu, \lambda}(\xi) \mathscr{F}[u](\xi)=\mathscr{F}[f](\xi)
$$

where

$$
\mathscr{B}_{p, q}^{\mu, \lambda}(\xi)=(-1)^{k+1}\left\{p \mathscr{B}_{+}^{\mu, \lambda}(\xi)+q \mathscr{B}_{-}^{\mu, \lambda}(\xi)\right\}
$$

and

$$
\mathscr{B}_{ \pm}^{\mu, \lambda}(\xi):= \begin{cases}(\lambda \pm \mathrm{i} \xi)^{\mu}-\lambda^{\mu}, & 0<\mu<1 \\ (\lambda \pm \mathrm{i} \xi)^{\mu}-\lambda^{\mu}- \pm \mathrm{i} \xi \mu \lambda^{\mu-1}, & 1<\mu<2 .\end{cases}
$$


Therefore, the solution can be found by applying the inverse Fourier transform to the term

$$
\mathscr{F}[u](\xi)=\frac{\mathscr{F}[f](\xi)}{1+\mathscr{B}_{p, q}^{\mu, \lambda}(\xi)} .
$$

Let $\left\{\xi_{j}=\widehat{x}_{j}\right\}_{j=0}^{N}$ be the set of Hermite-Gauss nodes. The Hermite collocation method consists in finding $u_{N} \in \widehat{\mathscr{P}}_{N}$ such that

$$
\mathscr{F}\left[u_{N}\right]\left(\xi_{j}\right)=\frac{\mathscr{F}\left[\mathscr{I}_{N} f\right]\left(\xi_{j}\right)}{1+\mathscr{B}_{p, q}^{\mu, \lambda}\left(\xi_{j}\right)}, \quad 0 \leq j \leq N,
$$

where $\mathscr{I}_{N}$ is the interpolation operator (2.3).

The numerical solution $u_{N}$ and $u_{N}\left(\widehat{x}_{j}\right), 0 \leq j \leq N$ can be determined by the following four steps:

1. Perform the forward discrete Hermite transform to obtain $\tilde{f}_{l}, 0 \leq l \leq N$ such that

$$
\mathscr{I}_{N} f(x)=\sum_{l=0}^{N} \tilde{f}_{l} \psi_{l}(x)
$$

2. Apply the Fourier transform to (4.3) and use Lemma 2.3 to obtain

$$
\mathscr{F}\left[\mathscr{I}_{N} f\right](\xi)=\sum_{l=0}^{N} \tilde{f}_{l}(-i)^{l} \psi_{l}(\xi)
$$

3. Determine $\mathscr{F}\left[u_{N}\right]\left(\xi_{j}\right), 0 \leq j \leq N$ in (4.2) and perform the forward Hermite transform to obtain $\tilde{u}_{l}, 0 \leq l \leq N$ such that

$$
\mathscr{F}\left[u_{N}\right](\xi)=\sum_{l=0}^{N} \tilde{u}_{l} \psi_{l}(\xi)
$$

4. Apply the inverse Fourier transform to (4.5) and obtain

$$
u_{N}(x)=\sum_{l=0}^{N} \tilde{u}_{l} i^{l} \psi_{l}(x)
$$

\subsection{Application to steady tempered fractional diffusion equations}

Consider the steady tempered fractional diffusion equation (4.1) with fixed parameters $p=q=1 / 2$ and $\lambda=5 / 2$. Applying (4.3)-(4.6) to (4.2), we can derive the numerical solution $u_{N}$.

Let $f(x)=(1+x) e^{-x^{2}}$. On the left of Fig. 4 , we plot the error curves in $L^{\infty}$-norm with $\mu=1.5$ and $\mu=0.8$, respectively. We note that the numerical solutions obtained by the proposed Hermite collocation method converge exponentially. We also consider algebraically decaying forcing function $f(x)=1 /\left(1+x^{2}\right)^{2}$ and numerical results displayed on the right of Fig. 4 demonstrate the algebraic convergence rate. Both numerical results verified the approximation theory of Hermite collocation method. Since the exact solutions are not known, we take $u_{N}, N=200$ as the reference solution in both cases. 

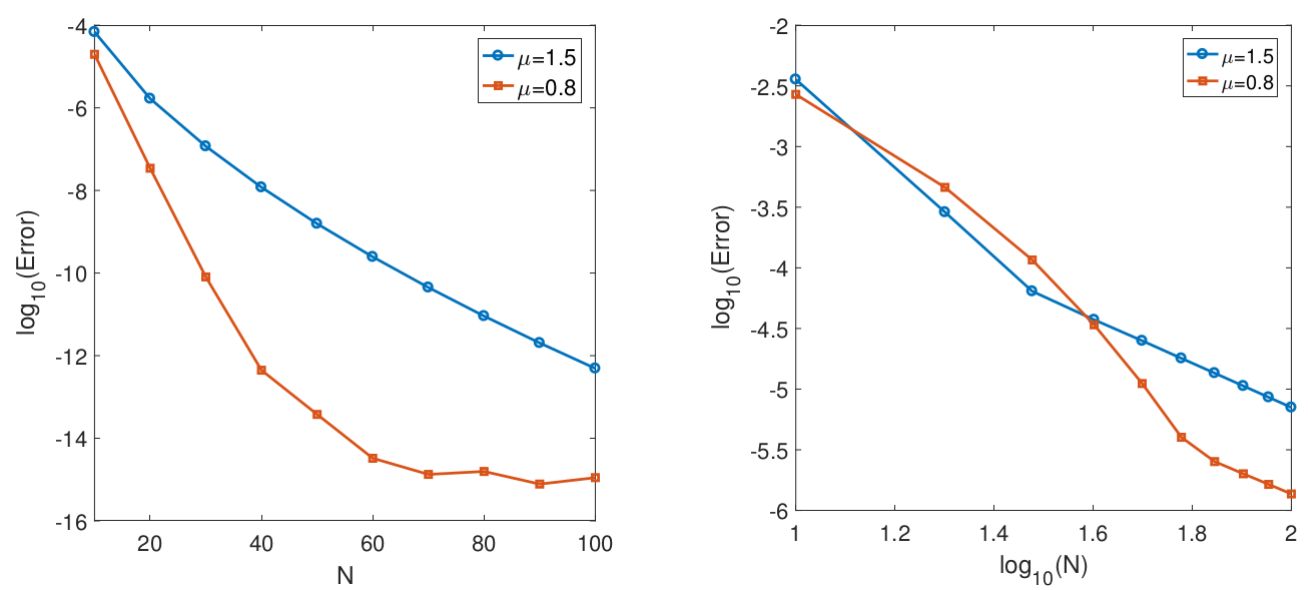

Figure 4: Left: $f(x)=(1+x) e^{-x^{2}}$. Right: $f(x)=1 /\left(1+x^{2}\right)^{2}$.

\subsection{Application to tempered fractional diffusion equations}

Using the same approach, we can numerically solve the tempered fractional diffusion equation (3.1). In fact, the application of the Fourier transform leads to the following equation:

$$
\begin{aligned}
& \mathscr{F}\left[\partial_{t} u\right](\xi, t)+\mathscr{B}_{p, q}^{\mu, \lambda}(\xi) \mathscr{F}[u](\xi, t)=\mathscr{F}[f](\xi, t), \\
& \mathscr{F}[u](\xi, 0)=\mathscr{F}\left[u_{0}\right](\xi) .
\end{aligned}
$$

The spectral collocation scheme is to solve $N+1$ ordinary differential equations

$$
\begin{aligned}
& \mathscr{F}\left[\partial_{t} u_{N}\right]\left(\xi_{j}, t\right)+\mathscr{B}_{p, q}^{\mu, \lambda}\left(\xi_{j}\right) \mathscr{F}\left[u_{N}\right]\left(\xi_{j}, t\right)=\mathscr{F}[f]\left(\xi_{j}, t\right), \\
& \mathscr{F}\left[u_{N}\right]\left(\xi_{j}, 0\right)=\mathscr{F}\left[u_{0}\right]\left(\xi_{j}\right), \quad j=0,1, \ldots, N .
\end{aligned}
$$

Analogously, we use the Crank-Nicolson scheme to solve the ordinary differential equations and derive the numerical solution $\mathscr{F}\left[u_{N}\right](\xi, t)$ for fixed $t=T$. Then, following (4.3)-(4.6), we can determine the solution $u_{N}^{\tau}(x, T)$ in the physical space.

- Consider the problem (3.1) with the exponentially decaying forcing term $f(x, t)=$ $\cos (t) e^{-x^{2}}$ and the initial distribution $u_{0}=10 e^{-4 x^{2}}$. On the left of Fig. 5, with fixed $p=q=1 / 2, \lambda=5 / 2, T=1$ and time step $\tau=0.001$, we show the errors in $L^{\infty}$. norm with $\mu=0.8$ and $\mu=1.7$, respectively. We observe that numerical solutions exponentially converge in the range of the time error $\tau^{2}$. In order to demonstrate the high-efficiency of the collocation method, distinct $\tau$, are chosen and the corresponding numerical results are presented on the right of Fig. 5. Since the exact solution is not known, we take $u=u_{N}^{\tau}, N=150, \tau=0.0001$ as the reference solution.

- Analogously to the steady case, the tempered fractional diffusion equation with algebraically decaying forcing term $f(x, t)$ is also considered. Keeping all sets of the 

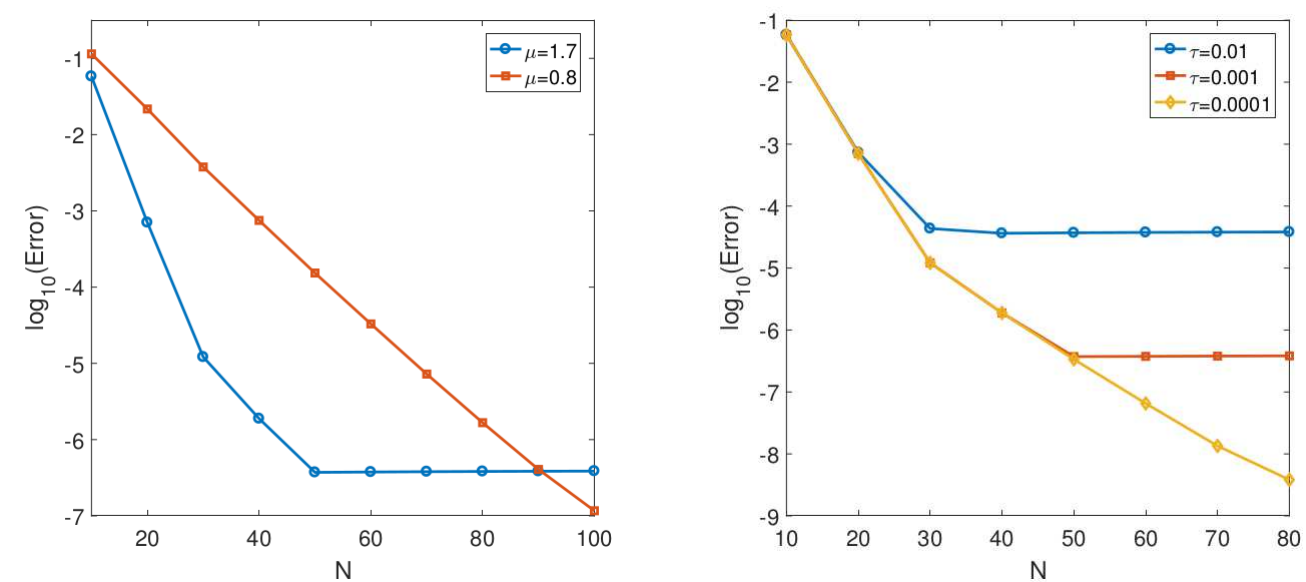

Figure 5: Collocation method for tempered fractional diffusion equation, $f(x, t)=\cos (t) e^{-x^{2}}, u_{0}=$ $10 e^{-4 x^{2}}, p=q=1 / 2, \lambda=5 / 2$.
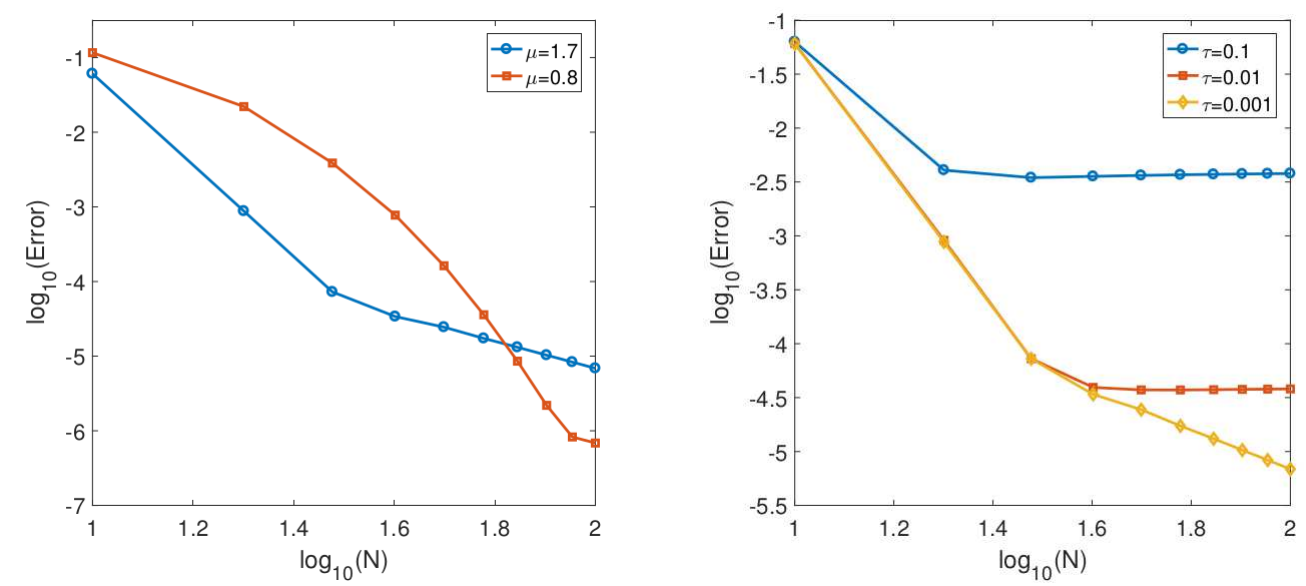

Figure 6: Collocation method for tempered fractional diffusion equation, $f(x, t)=\cos (t) /\left(1+x^{2}\right)^{2}$, $u_{0}=10 e^{-4 x^{2}}, p=q=1 / 2, \lambda=5 / 2$.

above case but taking $f(x, t)=\cos (t) /\left(1+x^{2}\right)^{2}$, we plot the error curves in Fig. 6 . The numerical results are consistent with theoretical findings. Numerical solutions converge with algebraic convergence rate.

\section{Conclusion}

Considering tempered fractional diffusion equations on the whole line, we develop spectral and spectral collocation methods based on the fact that Hermite functions are the eigenfunctions of the Fourier transform. Applying the Fourier transform to the underlying problem, we approximate the resulting equations by Hermite functions. The convergence 
and stability of such methods are analysed. Numerical examples demonstrate the efficiency of the algorithms and confirm theoretical findings. The approach is simple, efficient and accurate. It can be applied to more general problems with tempered fractional integrals and derivatives, including Riemann-Liouville tempered fractional integrals and derivatives defined in [36].

\section{Acknowledgments}

The second author is partially supported by the Natural Science Foundation of the Jiangsu Higher Education Institutions of China (Grant No. BK20181002), by the National Natural Science Foundation for the Youth of China (Grant No. 11801235), and by the Postdoctoral Science Foundation of China (Grant Nos. BX20180032, 2019M650459). The third author is supported in part by the NSFC (Grant Nos. 11771299, 11571151).

\section{References}

[1] T.M. Atanackovic and B. Stankovic, Generalized wave equation in nonlocal elasticity, Acta Mech. 208, 1-10 (2009).

[2] B. Baeumer, D.A. Benson, M.M. Meerschaert and S.W. Wheatcraft, Subordinated advectiondispersion equation for contaminant transport, Water Resour. Res. 37, 1543-1550 (2001).

[3] B. Baeumer, M. Kovacs and M.M. Meerschaert, Fractional reproduction-dispersal equations and heavy tail dispersal kernels, Bull. Math. Biol. 69(7), 2281-2297 (2007).

[4] B. Baeumer and M.M. Meerschaert, Tempered stable Lévy motion and transient super-diffusion, Comput. Appl. Math. 233(10), 2438-2448 (2010).

[5] Á. Carte and D. del-Castillo-Negrete, Fluid limit of the continuous-time random walk with general Lévy jump distribution functions, Phys. Rev. E 76 (2007).

[6] Á. Carte and D. del-Castillo-Negrete, Fractional diffusion models of option prices in markets with jumps, Phys. A 374(2), 749-763 (2007).

[7] C. Celik and M. Duman, Finite element method for a symmetric tempered fractional diffusion equation, Appl. Numer. Math. 120, 270-286 (2017).

[8] A.V. Chechkin, V.Yu. Gonchar, J. Klafter and R. Metzler, Natural cutoff in Lévy flights caused by dissipative nonlinearity, Phys. Rev. E 72010101 (2005).

[9] M.H. Chen and W.H. Deng, A second-order accurate numerical method for the space-time tempered fractional diffusion-wave equation, Appl. Math. Lett. 68, 87-93 (2017).

[10] S. Chen and J. Shen, An efficient and accurate numerical method for the spectral fractional Laplacian equation, J. Sci. Comput. 82(1), 1-25 (2020).

[11] S. Chen, J. Shen and L.L. Wang, Laguerre functions and their applications to tempered fractional differential equations on infinite intervals, J. Sci. Comput. 74, 1286-1313 (2018).

[12] S. Chen, J. Shen, Z.M. Zhang and Z. Zhou, A spectrally accurate approximation to subdiffusion equations using the log orthogonal functions, SIAM J. Sci. Comput. 42(2), A849-A877 (2020).

[13] J.H. Cushman and T.R. Ginn, Fractional advection-dispersion equation: a classical mass balance with convolution-Fickian flux, Water Resour. Res. 36(12), 3763-3766 (2000).

[14] M. Dehghan and M. Abbaszadeh, A finite difference/finite element technique with error estimate for space fractional tempered diffusion-wave equation, Comput. Math. Appl. 75, 2903-2914 (2018). 
[15] D. del-Castillo-Negrete, Truncation effects in superdiffusive front propaution with Lévy flights, Phys. Rev. E 79, 031120 (2009).

[16] W.H. Deng and Z.J. Zhang, Numerical schemes of the time tempered fractional Feynman-Kac equation, Comput. Math. Appl. 73, 1063-1076 (2017).

[17] Z.Q. Deng, L. Bengtsson and V.P. Singh, Parameter estimation for fractional dispersion model for rivers, Environ. Fluid. Mech. 6(5), 451-475 (2006).

[18] J. Duoandikoetxea, Fourier Analysis, Graduate Studies in Mathematics 29, AMS (2001).

[19] R. Gorenflo, F. Mainardi, E. Scalas and M. Raberto, Fractional calculus and continuous-time finance, Phys. A: Stat. Mech. Appl. 284, 376-384 (2000).

[20] E. Hanert and C. Piret, A Chebyshev pseudospectral method to solve the space-time tempered fractional diffusion equation, SIAM J. Sci. Comput. 36(4), A1797-A1812 (2014).

[21] J.H. Jeon, H.M. Monne, M. Javanainen and R. Metzler, Anomalous diffusion of phospholipids and cholesterols in a lipid bilayer and its origins, Phys. Rev. Lett. 109(18), 188103 (2012).

[22] J. Klafter, S.C. Lim and R. Metzler, Fractional Dynamics: Recent Advances, World Scientific (2011).

[23] I. Koponen, Analytic approach to the problem of convergence of truncated Lévy flights towards the Gaussian stochastic process, Phys. Rev. E 52(1), 1197-1198 (1995).

[24] C. Li and W.H. Deng, High order schemes for the tempered fractional diffusion equations, Adv. Comput. Math. 42, 543-572 (2016).

[25] M. Magdziarz, A. Weron and K. Weron, Fractional Fokker-Planck dynamics: stochastic representation and computer simulation, Phys. Rev. 75(2), 016708 (2007).

[26] B. Mandelbrot, Fractals and Scaling in Finance, Springer (1997).

[27] R.N. Mantegna and H.E. Stanley, Stochastic process with ultraslow convergence to a Gaussian: the truncated Lévy flight, Phys. Rev. Lett. 73(22), 2946-2949 (1994).

[28] Z.P. Mao and J. Shen, Hermite spectral methods for fractional PDEs in unbounded domains, SIAM J. Sci. Comput. 39(5), A1928-A1950 (2017).

[29] M.M. Meerschaert and E. Scalas, Coupled continuous time random walks in finance, Phys. A 370(1), 114-118 (2006).

[30] M.M. Meerschaert and A. Sikorskii, Stochastic Models for Fractional Calculus, Walter de Gruyter (2012).

[31] M.M. Meerschaert, Y. Zhang and B. Baeumer, Tempered anomalous diffusion in heterogeneous systems, Geophys. Res. Lett. 35, L17403 (2008).

[32] R. Metzler and J. Klafter, The random walk's guide to anomalous diffusion: a fractional dynamics approach, Phys. Rep. 339(1):1-77 (2000).

[33] R. Metzler and J. Klafter, The restaurant at the end of the random walk: recent developments in the description of anomalous transport by fractional dynamics, J. Phys. A 37, R161-R208 (2004).

[34] A. Piryatinska, A.I. Saichev and W.A. Woyczynski, Models of anomalous diffusion: the subdiffusive case, Phys. A 349(3), 375-420 (2005).

[35] J. Rosiński, Tempering stable processes, Stoch. Proc. Appl. 117, 667-707 (2007).

[36] F. Sabzikar, M.M. Meerschaert and J.H. Chen, Tempered fractional calculus, J. Comput. Phys. 293, 14-28 (2015).

[37] E. Scalas, Five years of continuous-time random walks in econophysics, in: Proceedings of WEHIA 2004, A. Namatame (Ed), (2004).

[38] J. Shen, T. Tang and L.L. Wang, Spectral Methods: Algorithms, Analysis And Applications, Springer-Verlag (2011).

[39] I.M. Sokolov, A.V. Chechkin and J. Klafter, Fractional diffusion equation for a power-lawtruncated Lévy process, Phys. A, 336(3), 245-251 (2004). 
[40] X.R. Sun, F.Q. Zhao and S.P. Chen, Numerical algorithms for the time-space tempered fractional Fokker-Planck equation, Adv. Difference Equ. 2017(1), 259 (2017).

[41] T. Tang, L.L. Wang, H.F. Yuan and T. Zhou, Rational spectral methods for PDEs involving fractional Laplacian in unbounded domains, SIAM J. Sci. Comput. 42(2), A585-A611 (2020).

[42] T. Tang, H.F. Yuan and T. Zhou, Hermite spectral collocation methods for fractional PDEs in unbounded domains, Commun. Comput. Phys. 24, 1143-1168 (2018).

[43] M. Zayernouri, M. Ainsworth and G.E. Karniadarkis, Tempered fractional Sturm-Liouville eigenproblems, SIAM J. Sci. Comput. 37(4), A1777-A1800 (2015).

[44] Y. Zhang, Moments for tempered fractional advection-diffusion equations, J. Stat. Phys. 139, 915-939 (2010). 